\title{
Per-Spectral Characterizations of Bicyclic Networks
}

\author{
Tingzeng $W u^{1}$ and Huazhong $L \ddot{u ̈}^{2}$ \\ ${ }^{1}$ School of Mathematics and Statistics, Qinghai Nationalities University, Xining, Qinghai 810007, China \\ ${ }^{2}$ School of Mathematics Science, University of Electronic Science and Technology of China, Chengdu, Sichuan 610054, China \\ Correspondence should be addressed to Tingzeng Wu; mathtzwu@163.com
}

Received 17 March 2017; Accepted 7 May 2017; Published 31 May 2017

Academic Editor: Zhi-Zhong Chen

Copyright (c) 2017 Tingzeng Wu and Huazhong Lü. This is an open access article distributed under the Creative Commons Attribution License, which permits unrestricted use, distribution, and reproduction in any medium, provided the original work is properly cited.

\begin{abstract}
Spectral techniques are used for the study of several network properties: community detection, bipartition, clustering, design of highly synchronizable networks, and so forth. In this paper, we investigate which kinds of bicyclic networks are determined by their per-spectra. We find that the permanental spectra cannot determine sandglass graphs in general. When we restrict our consideration to connected graphs or quadrangle-free graphs, sandglass graphs are determined by their permanental spectra. Furthermore, we construct countless pairs of per-cospectra bicyclic networks.
\end{abstract}

\section{Introduction}

It was recognized in about last decade that graph spectra have several important applications in computer science. Graph spectra appear in internet technologies, pattern recognition, computer vision, data mining, multiprocessor systems, statistical databases, and many other areas. For example, spectral filtering is applied in the study of Internet structure [1]. This method uses the eigenvectors of the adjacency and other graph matrices and some clusters in data sets represented by graphs. For more information about the applications of graph spectra in computer science see [2-6], among others.

The permanent of $n \times n$ matrix $Y=\left(y_{i j}\right)(i, j=1,2, \ldots, n)$ is defined as

$$
\operatorname{per}(Y)=\sum_{\sigma} \prod_{i=1}^{n} y_{i \sigma(i)}
$$

where the sum is taken over all permutations $\sigma$ of $\{1,2, \ldots, n\}$. Valiant [7] showed that computing the permanent is \#Pcomplete even when restricted to $(0,1)$-matrices.

Let $G$ be a graph with $n$ vertices, and let $A(G)$ be $(0,1)$ adjacency matrix of $G$. The permanental polynomial of $G$, denoted by $\pi(G, x)$, is defined as $\pi(G, x)=\operatorname{per}(x I-A(G))$. The permanental spectrum (per-spectrum for short) of graph $G$, denoted by $p s(G)$, is the set of all roots (together with their multiplicities) of $\pi(G, x)$.
Two graphs are per-cospectral if they share the same perspectrum. A graph $G$ is said to be determined by its perspectrum (DPS for short) if there is no other nonisomorphic graph with the same per-spectrum.

Which graphs are determined by their adjacency spectra is an old problem in graph spectra theory. van Dam and Haemers [8,9] gave an excellent survey of answers to the question of which graphs are determined by the spectra of some graph polynomials. Merris et al. [10] first considered the problem which graph is DPS. And they showed that the five pairs adjacency cospectral graphs (see [11]) are DPS. Based on the result, they formulated that the per-spectrum seems a little better than the adjacency spectrum when it comes to distinguishing graphs which are not trees. In fact, characterizing what kinds of graphs are determined by the per-spectra is generally a very hard problem. Up to now, only a few types of graphs are proved to be DPS; see [12-17].

A bicyclic network is a simple connected graph in which the number of edges equals the number of vertices plus one [18]. The sandglass graph is a bicyclic network, denoted by $S\left(C_{3}, P_{h}, C_{3}\right)$, obtained by appending a triangle to each pendant vertex of the path $P_{h}$. Lu et al. [19] proved that sandglass graphs are determined by their adjacency spectra. Motivated by the statement of Merris et al., a natural problem is whether sandglass graphs are determined by their perspectra. In this paper, we give a solution of this question. 


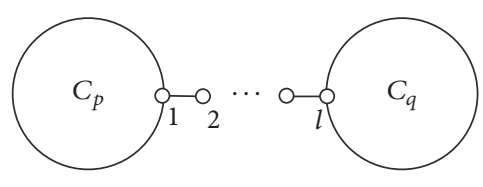

$\infty(p, l, q)$

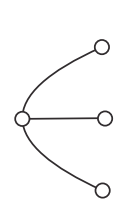

$\theta(r, s, t)$

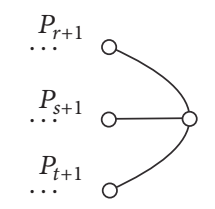

FIGURE 1: $\infty$-graph and $\theta$-graph.
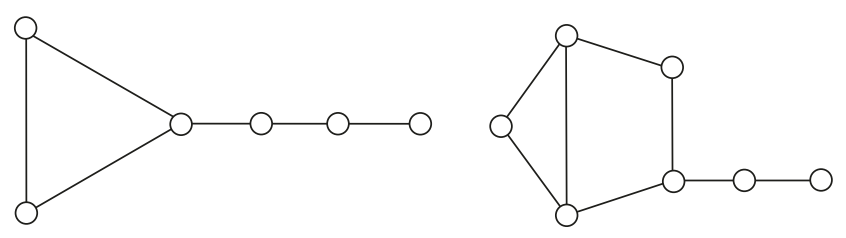

Figure 2: Graph $G^{\prime}$.

Lemma 3 (see [17]). Let $G$ be a graph with $m$ edges, and let $t_{i}(G)$ denote the degree sum of the three vertices on ith triangle in $G$. Then

$$
b_{5}(G)=-2\left(\sum_{i=1}^{c_{3}(G)}\left(m+3-t_{i}(G)\right)+c_{5}(G)\right) .
$$

Lemma 4 (see [13]). The following can be deduced from the permanental polynomial of a graph $G$ :

(i) The number of vertices

(ii) The number of edges

(iii) The number of triangles

(iv) The length of the shortest odd cycle

(v) The number of the shortest odd cycles

(vi) Whether $G$ is bipartite

\section{Sandglass Graphs Are DPS}

In this section, we will give the solutions of the problem which sandglass graphs are DPS?

Checking graph $G^{\prime}$ depicted in Figure 2, direct computation yields $\pi\left(G^{\prime}, x\right)=x^{13}+14 x^{11}-4 x^{10}+74 x^{9}-40 x^{8}+$ $186 x^{7}-136 x^{6}+230 x^{5}-180 x^{4}+130 x^{3}-76 x^{2}+25 x-4=$ $\pi\left(S\left(C_{3}, P_{9}, C_{3}\right), x\right)$. This implies that the permanental spectra cannot determine sandglass graphs in general. Examining graph $G^{\prime}$ again, we know that $G^{\prime}$ is not connected and contains a quadrangle. It is natural to consider the problem which sandglass graphs are DPS when we restrict our consideration to connected graphs or quadrangle-free graphs, where the quadrangle-free graph is one which contains no quadrangles (i.e., cycles of length 4). We will answer these questions one by one in the following.

First, we give some lemmas which will play an important role in the proof of main theorems.

Lemma 5. Let $S\left(C_{3}, P_{h}, C_{3}\right)$ be a graph with $n$ vertices. Then $b_{5}\left(S\left(C_{3}, P_{h}, C_{3}\right)\right)=-4 n+12$.

Proof. By Lemma 3, the proof is trivial.

Lemma 6. Let $S\left(C_{3}, P_{h}, C_{3}\right)$ be a graph with $n$ vertices. Then $n$-th coefficient of $\pi\left(S\left(C_{3}, P_{h}, C_{3}\right), x\right)$ is

$$
b_{n}\left(S\left(C_{3}, P_{h}, C_{3}\right)\right)= \begin{cases}-4, & \text { if } n \text { is odd } \\ 5, & \text { if } n \text { is even }\end{cases}
$$


Proof. Suppose that $n$ is odd. Then the Sachs subgraph of order $n$ in $S\left(C_{3}, P_{h}, C_{3}\right)$ is only $C_{3} \cup((n-3) / 2) P_{2}$. By Lemma 1 , we have $b_{n}(G)=-4$. Otherwise, if $n$ is even, then the Sachs subgraph of order $n$ in $S\left(C_{3}, P_{h}, C_{3}\right)$ is $C_{3} \cup C_{3} \cup((n-6) / 2) P_{2}$ and $(n / 2) P_{2}$. By Lemma 1 , we have $b_{n}(G)=5$.

Lemma 7. Let $G$ be a quadrangle-free graph with $n$ vertices. If $\pi(G, x)=\pi\left(S\left(C_{3}, P_{h}, C_{3}\right), x\right)$, then the degree sequence of $G$ is $\left(2^{n-2}, 3^{2}\right)$, where $a^{k}$ means $\overbrace{a, a, \ldots, a}^{k}$.

Proof. Suppose that the degree sequence $G$ is $\left(3+t_{1}, 3+t_{2}, 2+\right.$ $\left.t_{3}, \ldots, 2+t_{n}\right)$, where $t_{1}, t_{2}>-3$, and $t_{i}>-2(i=3,4, \ldots, n)$ are integers. Since $G$ and $S\left(C_{3}, P_{h}, C_{3}\right)$ have the same number of edges, then

$$
\sum_{i=1}^{n} t_{i}=0
$$

By Lemma 2, we have

$$
\begin{aligned}
b_{4}(G)= & \left(\begin{array}{c}
n+1 \\
2
\end{array}\right)-\left(\begin{array}{c}
3+t_{1} \\
2
\end{array}\right)-\left(\begin{array}{c}
3+t_{2} \\
2
\end{array}\right) \\
& -\sum_{i=3}^{n}\left(\begin{array}{c}
2+t_{i} \\
2
\end{array}\right) .
\end{aligned}
$$

Since $\pi(G, x)=\pi\left(S\left(C_{3}, P_{h}, C_{3}\right), x\right)$, we have $b_{4}(G)=$ $b_{4}\left(S\left(C_{3}, P_{h}, C_{3}\right)\right)$. By a simple calculation, we obtain

$$
\sum_{i=1}^{n} t_{i}^{2}=-2\left(t_{1}+t_{2}\right) .
$$

Checking (8), it is easy to see that if $\left|t_{1}\right| \geq 3$ or $\left|t_{2}\right| \geq 3$, then $\sum_{i=3}^{n} t_{i}^{2}<0$, a contradiction. Hence,

$$
-2 \leq t_{1}, t_{2} \leq 2 .
$$

Furthermore, if $t_{1}=-t_{2}$ except $t_{1}=t_{2}=0$, then $\sum_{i=3}^{n} t_{i}^{2}<0$, a contradiction. Thus

$$
t_{1}+t_{2} \neq 0 \text {. }
$$

Solving simultaneous equations (6)-(10), we have

$$
\begin{aligned}
& t_{1}=t_{2}=-2, \\
& t_{3}=\cdots=t_{n}=0, \\
& t_{1}=-2, \\
& t_{2}=-1, \\
& t_{3}= \pm 1, \\
& t_{4}=\cdots=t_{n}=0, \\
& t_{1}=-2, \\
& t_{2}=0, \\
& t_{3}=\cdots=t_{n}=0, \\
& t_{1}=t_{2}=-1, \\
& t_{3}=t_{4}= \pm 1,
\end{aligned}
$$

$$
\begin{aligned}
& t_{5}=\cdots=t_{n}=0, \\
& t_{1}=-1, \\
& t_{2}=0, \\
& t_{3}= \pm 1, \\
& t_{4}=\cdots=t_{n}=0, \\
& t_{1}=0, \\
& t_{2}=-2, \\
& t_{3}=\cdots=t_{n}=0, \\
& t_{1}=0, \\
& t_{2}=-1, \\
& t_{3}= \pm 1, \\
& t_{4}=\cdots=t_{n}=0, \\
& t_{1}=t_{2}=0, \\
& t_{3}=\cdots=t_{n}=0 .
\end{aligned}
$$

Thus the degree sequence of $G$ is possible $\left(1^{2}, 2^{n-2}\right)$, $\left(1,2^{n-2}, 3\right)$, or $\left(2^{n-2}, 3^{2}\right)$. It is not difficult to check that only $\left(2^{n-2}, 3^{2}\right)$ satisfies the well-known hand-shaking theorem. So, the degree sequence of $G$ is $\left(2^{n-2}, 3^{2}\right)$.

Theorem 8. Restricting consideration on quadrangle-free graphs, sandglass graphs are determined by their per-spectra.

Proof. Let $G$ be a quadrangle-free graph with $n$ vertices percospectral with $S\left(C_{3}, P_{h}, C_{3}\right)$. By Lemma 7 , we know that the degree sequence of $G$ is $\left(2^{n-2}, 3^{2}\right)$. Then $G$ is isomorphic to $\infty(p, l, q) \cup\left(\bigcup_{i=1}^{k} C_{k_{i}}\right)$ or $\theta(r, s, t) \cup\left(\bigcup_{i=1}^{k} C_{k_{i}}\right)$, where $\bigcup_{i=1}^{k} C_{k_{i}}$ denotes the union of $k \geq 0$ disjoint cycles $C_{k_{i}}$ of length $k_{i}$. By the above, it implies that $|V(G)| \geq 5$. It is not difficult to see that if $n=5$, then $G$ is isomorphic to $S\left(C_{3}, P_{l}, C_{3}\right)$. So, assume $n>5$ and consider the following two cases.

Case 1. Assume that $G$ is isomorphic to $\infty(p, l, q) \cup\left(\bigcup_{i=1}^{k} C_{k_{i}}\right)$. We further discuss the following three subcases.

Subcase 1.1. Exact one of the two triangles belongs to the bicyclic component. Then $G=\infty(p, l, q) \cup C_{3} \cup \cup_{i=1}^{k-1} C_{k_{i}}$ for $p=3, q \geq 4$, and $k \geq 1$. By Lemma 3, we obtain that $b_{5}(G)=-4 n+10-2 c_{5}(G)$. By Lemma 5 , we have $b_{5}\left(S\left(C_{3}, P_{l}, C_{3}\right)\right)-b_{5}(G)=2+2 c_{5}(G)>0$. This contradicts the assumption that $G$ and $S\left(C_{3}, P_{l}, C_{3}\right)$ are per-cospectral.

Subcase 1.2. Both of the two triangles belong to the bicyclic component. Then $G=\infty(3, l, 3) \cup \bigcup_{i=1}^{k} C_{k_{i}}$. If $k=0$, then $G$ is isomorphic to $S\left(C_{3}, P_{l}, C_{3}\right)$. Next we suppose that $k>0$. By the structure of $G$ and Lemma 1 , we can obtain that $b_{n}(G)>5$ when $n$ is even, and $b_{n}(G)<-4$ when $n$ is odd. By Lemma 6, this is a contradiction. 
Subcase 1.3. Neither of the two triangles belongs to the bicyclic component. Then $G=\infty(p, l, q) \cup C_{3} \cup C_{3} \cup \bigcup_{i=1}^{k-2} C_{k_{i}}$ for $p, q \geq 4$. By Lemma 3, we have $b_{5}(G)=-4 n+8-2 c_{5}(G)$. So, $b_{5}\left(S\left(C_{3}, P_{l}, C_{3}\right)\right)-b_{5}(G)=4+2 c_{5}(G)>0$. This contradicts the assumption that $G$ and $S\left(C_{3}, P_{l}, C_{3}\right)$ are per-cospectral.

Case 2. Assume that $G$ is isomorphic to $\theta(r, s, t) \cup\left(\bigcup_{i=1}^{k} C_{k_{i}}\right)$. We further consider the following three subcases.

Subcase 2.1. Exact one of the two triangles belongs to the bicyclic component. Then $G=\theta(1,2, t) \cup C_{3} \cup \bigcup_{i=1}^{k-1} C_{k_{i}}$ for $t>2$ and $k \geq 1$. By the structure of $G$ and Lemma 1 , we can obtain that if $n$ is even, then $b_{n}(G)>5$; and if $n$ is odd, then $b_{n}(G)<-4$. By Lemma 6 , this is a contradiction.

Subcase 2.2. Both of the two triangles belong to the bicyclic component. Then $G=B_{2}(1,2,2) \cup\left(\bigcup_{i=1}^{k} C_{k_{i}}\right)$, which is a contradiction to $G$ being a quadrual-free graph.

Subcase 2.3. Neither of the two triangles belongs to the bicyclic component. Then $G=\theta(r, s, t) \cup C_{3} \cup C_{3} \cup\left(\bigcup_{i=1}^{k-2} C_{k_{i}}\right)$ for $r>1, s, t \geq 2$ or $r \geq 1, s, t>2$. By Lemma 3, we have $b_{5}(G)=-4 n+8-2 c_{5}(G)$. By Lemma 6 , we have a contradiction, and the theorem follows.

Theorem 9. Restricting consideration on connected graphs, sandglass graphs are determined by their per-spectra.

Proof. Let $G$ be a connected graph, where $|G|=n$ and $n \geq 5$, and let $G$ be per-cospectral with $S\left(C_{3}, P_{h}, C_{3}\right)$. By Lemma $4, G$ is a bicyclic graph with two triangles and must be isomorphic to a graph containing a sandglass graph $S\left(C_{3}, P_{l}, C_{3}\right)$ as its induced subgraph or $K_{4}-e$ (isomorphic to $\theta$-graph when $r=1$ and $s=t=2$ ) as its induced subgraph.

Suppose that $G$ is isomorphic to a graph which contains a sandglass graph $S\left(C_{3}, P_{l}, C_{3}\right)$ as its induced subgraph. Then $G$ contains no quadrangles. By Lemma 7, $G$ must be isomorphic to the sandglass graph $S\left(C_{3}, P_{h}, C_{3}\right)$.

In the following, we will prove that $G$ is isomorphic to a graph containing $K_{4}-e$ as its induced subgraph.

Suppose that $n$ is even. By Lemma 6, we know that $b_{n}\left(S\left(C_{3}, P_{h}, C_{3}\right)\right)=5$. This implies, by Lemma 1 , that $G$ must have odd perfect matchings. Examining the structure of $G$, we see that $G$ has at most two perfect matchings. So, $G$ only has uniquely one perfect matching. This implies that all triangles or 4-cycle in $G$ are not a component of some Sachs subgraph of order $n$. Thus, the perfect matching of $G$ is a unique Sachs subgraph of order $n$. By Lemma $1, b_{n}(G)=1$, which contradicts the fact that $b_{n}\left(S\left(C_{3}, P_{h}, C_{3}\right)\right)=5$.

Assume that $n$ is odd. By Lemma 1 and examining the structure of $G$, we know that the Sachs subgraphs of order $n$ in $G$ is only the union of a triangle and a perfect matching of $G$ deleting all edges on the triangle. Then $b_{n}(G)=2$. This contradicts $b_{n}\left(S\left(C_{3}, P_{h}, C_{3}\right)\right)=5$.

This completes the proof.

For any bicyclic network, it is difficult to discuss which is determined by its per-spectrum. We can construct countless pairs per-cospectral bicyclic networks. Let $H$ be an arbitrary

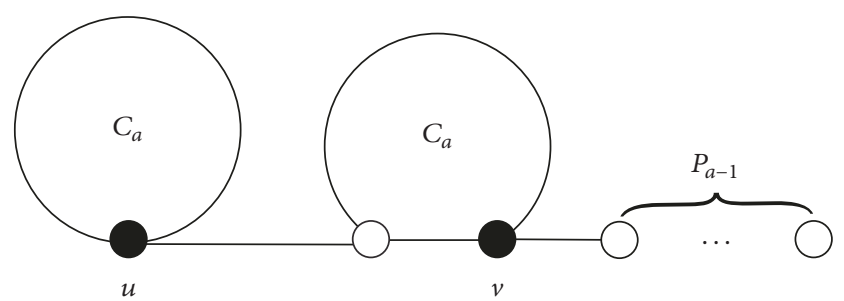

Figure 3: The bicyclic network $B$.

graph with a fixed vertex $w$ and let $G_{u} \cdot H$ denote the coalescence of $G$ and $H$ with respect to $u$ and $w$, which is the graph obtained from $G \cup H$ by identifying $u$ and $w$. Similarly, we define $G_{v}^{\prime} \cdot H$. Borowiecki [20] showed that if both $G-u$ and $G^{\prime}-v$ are per-cospectral, then both $G_{u} \cdot H$ and $G_{v}^{\prime} \cdot H$ are also per-cospectral. As an example, let $B=B^{\prime}$ be the bicyclic network depicted in Figure 3. As $B-u$ and $B-v$ are isomorphic, they are per-cospectral. By the above-mentioned result of Borowiecki [20], for any graph $H$, both $B_{u} \cdot H$ and $B_{v} \cdot H$ are per-cospectral.

\section{Summary}

Per-spectra is an important part of graph spectra. In this paper, we discuss properties of permanental spectra of bicyclic networks. We show that without some limitations bicyclic networks are not DPS. Particularly, we find a pair of per-cospectral graphs. Combining the result of $\mathrm{Lu}$ et al. [19], our results (Theorems 8 and 9) are beyond Merris et al.'s imagination. Finally, we pose the following conjecture.

Conjecture 10. Sandglass graphs with a perfect matching are DPS.

\section{Conflicts of Interest}

The authors declare that they have no conflicts of interest.

\section{Acknowledgments}

The authors thank Dr. Shunyi Liu for providing Figure 2. The authors are supported by NSF of Qinghai (2016-ZJ-947Q) and High-level personnel of scientific research projects of QHMU(2016XJG07).

\section{References}

[1] C. Gkantsidis, M. Mihail, and E. Zegura, "Spectral analysis of internet topologies," in Proceedings of Twenty-Second Annual Joint Conference of the IEEE Computer and Communications (INFOCOM '03), vol. 1, pp. 364-374, San Francisco, Calif, USA, 2003.

[2] D. Cvetkovic' and T. Davidovic', "Multiprocessor interconnection networks," in Applications of Graph Spectra, Zbornik radova 13(21), D. Cvetkovic' and I. Gutman, Eds., vol. 13, pp. 33-63, Mathematical Institute SANU, Belgrade, Serbia, 2009.

[3] D. Cvetkovic', "Applications of Graph Spectra," in An introduction to the literature, Applications of Graph Spectra, Zbornik 
radova 13(21), D. Cvetkovic' and I. Gutman, Eds., pp. 7-31, Mathematical Institute SANU, Belgrade, Serbia, 2009.

[4] F. Chung and L. Lu, Complex Graphs and Networks, American Mathematical Society, Providence, Rhode Island, 2006.

[5] U. von Luxburg, "A tutorial on spectral clustering," Statistics and Computing, vol. 17, no. 4, pp. 395-416, 2007.

[6] P. V. Mieghem, "Graph spectra for complex networks," Graph Spectra for Complex Networks, pp. 1-346, 2010.

[7] L. G. Valiant, "The complexity of computing the permanent," Theoretical Computer Science, vol. 8, no. 2, pp. 189-201, 1979.

[8] E. R. van Dam and W. H. Haemers, "Which graphs are determined by their spectrum?" Linear Algebra and its Applications, vol. 373, pp. 241-272, 2003.

[9] E. R. van Dam and W. H. Haemers, "Developments on spectral characterizations of graphs," Discrete Mathematics, vol. 309, no. 3, pp. 576-586, 2009.

[10] R. Merris, K. R. Rebman, and W. Watkins, "Permanental polynomials of graphs," Linear Algebra and its Applications, vol. 38, pp. 273-288, 1981.

[11] F. Harary, C. King, A. Mowshowitz, and R. C. Read, "Cospectral graphs and digraphs," The Bulletin of the London Mathematical Society, vol. 3, pp. 321-328, 1971.

[12] W. Li, S. Liu, T. Wu, and H. Zhang, "On the permanental polynomials of graphs," in Graph Polynomials, Y. Shi and et al., Eds., CRC Press, 2016.

[13] S. Liu and H. Zhang, "On the characterizing properties of the permanental polynomials of graphs," Linear Algebra and its Applications, vol. 438, no. 1, pp. 157-172, 2013.

[14] T. Wu and H. Zhang, "Per-spectral characterizations of graphs with extremal per-nullity," Linear Algebra and its Applications, vol. 484, Article ID 13254, pp. 13-26, 2015.

[15] T. Wu and H. Zhang, "Per-spectral and adjacency spectral characterizations of a complete graph removing six edges," Discrete Applied Mathematics. The Journal of Combinatorial Algorithms, Informatics and Computational Sciences, vol. 203, pp. 158-170, 2016.

[16] T. Wu and H. Zhang, "Per-spectral characterizations of some bipartite graphs," Discussiones Mathematicae Graph Theory, 2017.

[17] H. Zhang, T. Wu, and H.-J. Lai, "Per-spectral characterizations of some edge-deleted subgraphs of a complete graph," Linear and Multilinear Algebra, vol. 63, no. 2, pp. 397-410, 2015.

[18] J. Ma, Y. Shi, Z. Wang, and J. Yue, "On Wiener polarity index of bicyclic networks," Scientific Reports, vol. 6, Article ID 19066, 2016.

[19] P. Lu, X. Liu, Z. Yuan, and X. Yong, "Spectral characterizations of sandglass graphs," Applied Mathematics Letters. An International Journal of Rapid Publication, vol. 22, no. 8, pp. 1225-1230, 2009.

[20] M. Borowiecki, "On spectrum and per-spectrum of graphs," Publications de l'Institut Mathématique, vol. 38, pp. 31-33, 1985. 


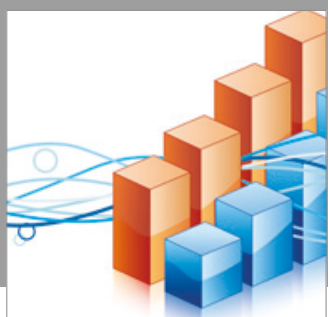

Advances in

Operations Research

vatersals

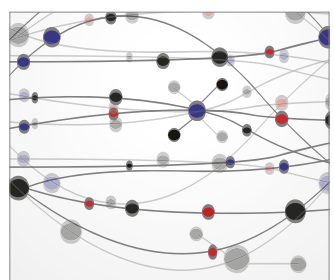

\section{The Scientific} World Journal
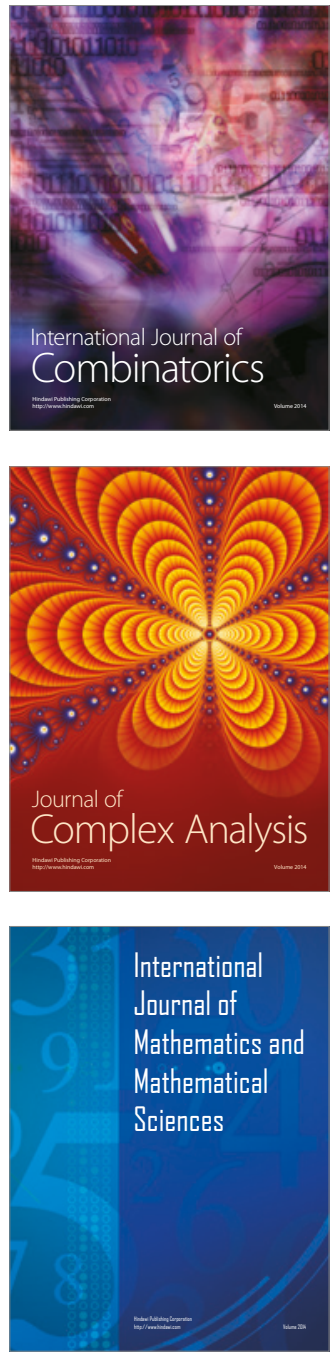
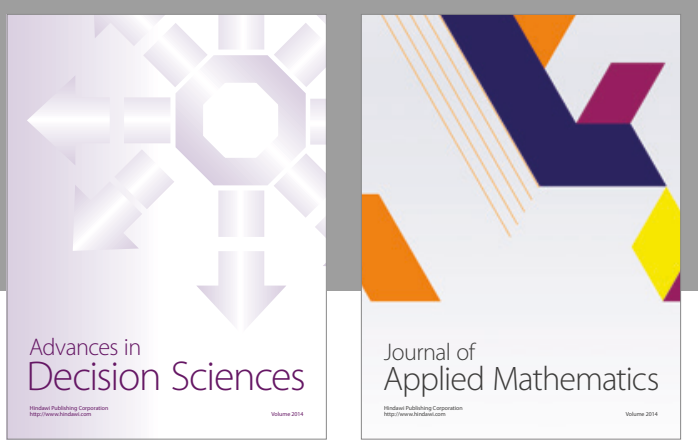

Algebra

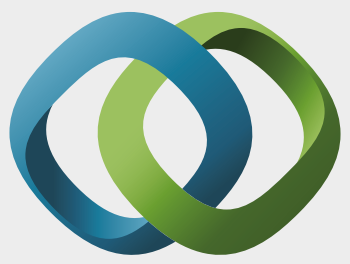

\section{Hindawi}

Submit your manuscripts at

https://www.hindawi.com
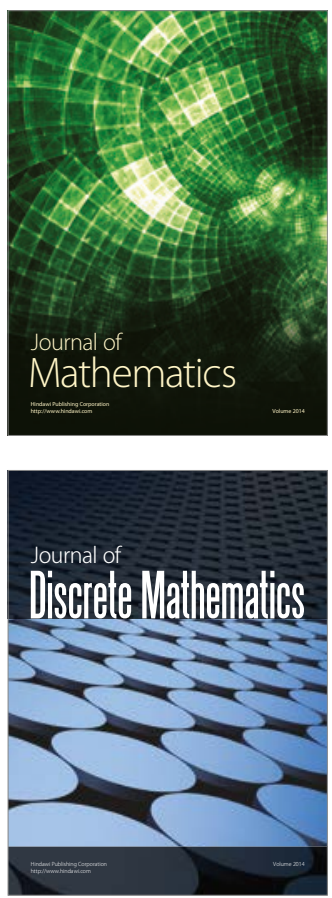

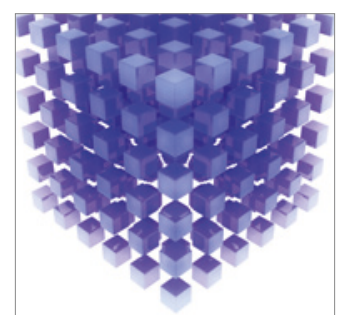

Mathematical Problems in Engineering
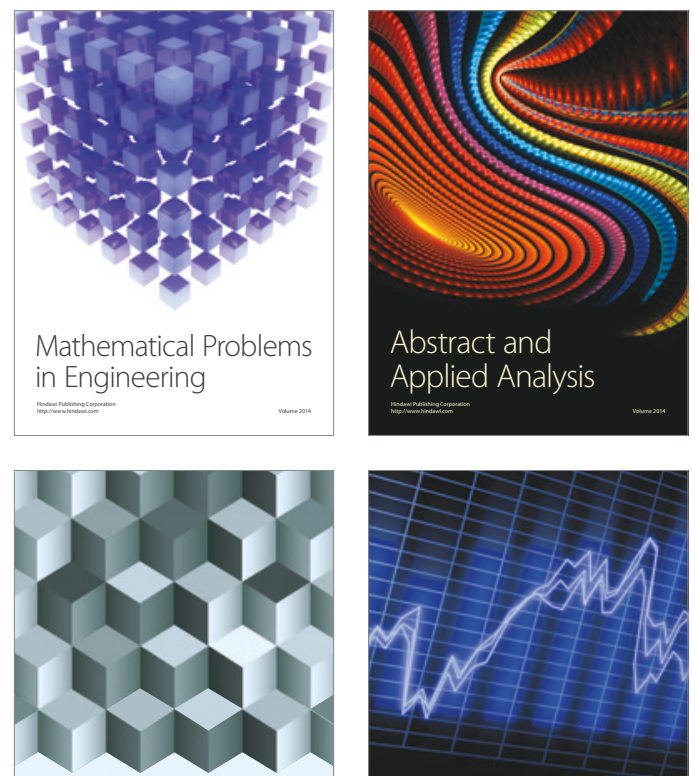

Journal of

Function Spaces

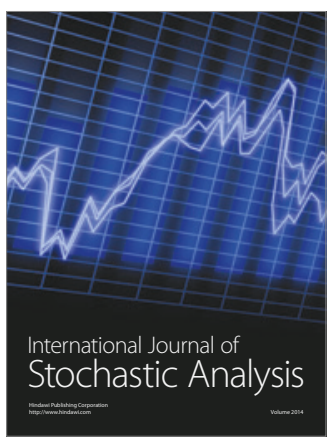

Probability and Statistics
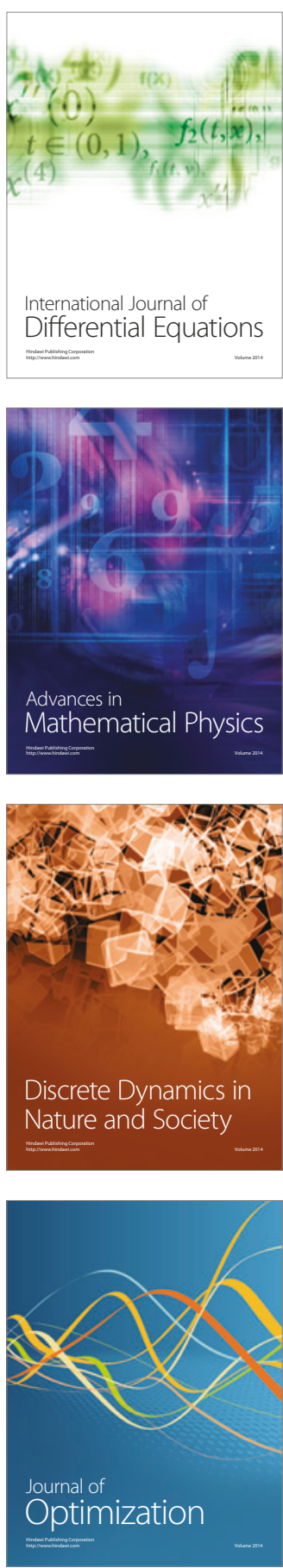